() Open Access Full Text Article

REVIEW

\title{
Increased Metabolic Burden Among Blacks: A Putative Mechanism for Disparate COVID-19 Outcomes
}

This article was published in the following Dove Press journal: Diabetes, Metabolic Syndrome and Obesity: Targets and Therapy

\author{
Girardin Jean-Louis (D) \\ Arlener D Turner (iD) \\ Peng Jin ${ }^{2}$ \\ Mengling $\mathrm{Liu}^{2}$ \\ Carla Boutin-Foster ${ }^{3}$ \\ Samy I McFarlane (iD ${ }^{3}$ \\ Azizi Seixas'
}

'Departments of Population Health, Psychiatry, and Biostatistics, New York University Langone Health, New York, NY, USA; ${ }^{2}$ Department of Biostatistics, New York University Langone Health, New York, NY, USA; ${ }^{3}$ Departments of Medicine and Endocrinology, SUNY Downstate Medical Center, New York, NY, USA
Correspondence: Girardin Jean-Louis NYU Grossman School of Medicine, 180 Madison Avenue, 7th Floor, New York, NY 10016, USA

$\mathrm{Tel}+$ | 646.50I.343 I

Email Girardin.Jean-Louis@nyulangone. org

\begin{abstract}
Mounting evidence shows a disproportionate COVID-19 burden among Blacks. Early findings indicate pre-existing metabolic burden (eg, obesity, hypertension and diabetes) as key drivers of COVID-19 severity. Since Blacks exhibit higher prevalence of metabolic burden, we examined the influence of metabolic syndrome on disparate COVID-19 burden. We analyzed data from a NIH-funded study to characterize metabolic burden among Blacks in New York (Metabolic Syndrome Outcome Study). Patients ( $\mathrm{n}=1035)$ were recruited from outpatient clinics, where clinical and self-report data were obtained. The vast majority of the sample was overweight/obese (90\%); diagnosed with hypertension (93\%); dyslipidemia $(72 \%)$; diabetes $(61 \%)$; and nearly half of them were at risk for sleep apnea $(48 \%)$. Older Blacks (age $\geq 65$ years) were characterized by higher levels of metabolic burden and comorbidities (eg, heart disease, cancer). In multivariate-adjusted regression analyses, age was a significant $(\mathrm{p} \leq .001)$ independent predictor of hypertension $(\mathrm{OR}=1.06 ; 95 \% \mathrm{CI}: 1.04-1.09)$, diabetes (OR=1.03; 95\% CI: 1.02-1.04), and dyslipidemia (OR=0.98; 95\% CI: $0.97-0.99)$, but not obesity. Our study demonstrates an overwhelmingly high prevalence of the metabolic risk factors related to COVID-19 among Blacks in New York, highlighting disparate metabolic burden among Blacks as a possible mechanism conferring the greater burden of COVID-19 infection and mortality represented in published data.
\end{abstract}

Keywords: COVID-19 burden, blacks, metabolic syndrome, metabolic risk factors, disparities

\section{Introduction}

Recent observations indicate that individuals in minority communities are disproportionately affected by the adverse effects of the COVID-19 pandemic. ${ }^{1,2}$ Data from the Center for Disease Control revealed that, although Blacks only comprise $13 \%$ of the US population, $33 \%$ of individuals requiring hospitalization due to COVID-19 were Black. ${ }^{3}$ Evidence also shows the COVID-19 fatality rate among Blacks is $19.8 \%$, which nearly doubles the rate observed among their white counterparts $(10.2 \%){ }^{2}$ Within NYC, an important epicenter of COVID-19, Blacks comprise only $9 \%$ of the population; yet, as of May 11, 2020 they had the highest death rate estimated at 217 per 100,000 people. ${ }^{4}$ These particular health disparities are not unprecedented, as similar inequities exist across other health conditions, likely undergirded by recalcitrant structural health inequities. ${ }^{5}$ In fact, the extant literature is replete with urgent calls to implement health reforms that will dismantle structural factors underpinning health inequities in black communities. ${ }^{6}$ Although no 
incontrovertible evidence has been proffered to date that COVID-19 would preferentially propagate in diverse communities, the unacceptably high number of COVID-related fatalities demands a systematic inquiry as to why such a large distribution of these fatalities have occurred in Black communities. $^{7-9}$

Early reports have demonstrated that individuals with underlying cardiometabolic diseases appear to be at greatest risk for COVID-19 infection and COVID-19-related mortality. ${ }^{1}$ Of interest, epidemiologic data revealed increased mortality risks seem to exceed the contributory effects of other known risk factors including socioeconomic position, a history of smoking and/or alcohol consumption. ${ }^{2}$ It is noteworthy that diseases that comprise the metabolic syndrome feature most prominently among the medical conditions that exacerbate the COVID-19 disease burden. Metabolic syndrome is a cluster of diseases (obesity, hypertension, dyslipidemia, diabetes) that substantially increases risk for morbidity and early mortality. Unfortunately, while the syndrome is prevalent in the United States overall, the highest burden is experienced in Black communities. ${ }^{10,11}$ This is compounded by the observation that social determinants of health (eg, socioeconomic disadvantage or economic deprivation), which are more common in diverse communities, play an important role in the elevated metabolic syndrome burden of Blacks. ${ }^{12}$ It may be that the presence of metabolic syndrome as well as the observed social determinants of health place Blacks at an elevated risk of COVID-19 burden.

Discussions regarding the reduction of disparate health burden in the Black community tend to focus initially on the multiple structural inequities in current health systems, which have long plagued diverse communities. ${ }^{5}$ In that regard, it is critical that public health experts acknowledge system-level factors undergirding health inequities when considering the implementation of new programs to flatten the curve of COVID-19 infections and fatalities in underserved communities. A critical evaluation of these systemlevel factors that reduce adequate access to care need to be addressed urgently, given the ballooning prevalence of COVID-19 infection and fatality among blacks. Evidence linking increased age and pre-existing cardiometabolic conditions (eg, obesity, hypertension, dyslipidemia, diabetes, and heart disease) with increased burden of COVID-19 infection, disease severity, and mortality seem to provide an opportunity to develop insightful and targeted strategies to reduce the burden of COVID-19 among blacks.

In this investigation, we analyzed data emanated from the Metabolic Syndrome Outcome Study (MetSO), ${ }^{13}$ an
NIH-funded study providing a unique opportunity to characterize Blacks in New York, a group that has, to date experienced $28 \%$ of COVID-related fatalities. ${ }^{2}$ In addition to the data on metabolic conditions, MetSO also enables the assessment of the contributory role of sociodemographic, psychosocial factors and health risk (eg, smoking, drinking, and sleep-disordered breathing) commonly associated with metabolic burden among Blacks in Brooklyn, NY. ${ }^{13-16}$ The objective of this study is to explore the burden of metabolic syndrome in a Black community within the epicenter of the COVID-19 pandemic in order to shed light on the disproportionate case fatalities in Black communities.

\section{Methods}

\section{Study Population}

The present analysis was based on data obtained from the Metabolic Syndrome Outcome (MetSO) study, an NIHfunded study of Non-Hispanic Blacks with metabolic syndrome. ${ }^{13,14}$ Participants were recruited from four outpatient clinics associated with SUNY Downstate Medical Center: Family Medicine Clinic, Heart Failure Clinic, Diabetes Clinic, and Lefferts Community Health Center, between 2009 and 2012. Inclusion criteria were adults ( $\geq 18$-years-old) with self-reported race/ethnicity as black/ African American with the diagnosis of metabolic syndrome. A total of 1035 patients agreed to participate in the study, 30 participants were excluded because of lack of valid data, which included missing information for sociodemographic factors, health risks, medical history, and/or psychological health. Physician-diagnosed conditions were derived from a query of an electronic medical record system (HealthBridge regional health information clearing-house) at SUNY Downstate Medical Center, a designated Brooklyn-based COVID-19 hospital. HealthBridge is a HIPAA-compliant record management system/data distribution network with a user-friendly interface allowing access to health-care data. The study was approved by the institutional review boards at NYU Langone and SUNY Downstate Medical Centers, all procedures were performed in accordance with the ethical standards and all patients provided informed consent prior to study enrollment.

\section{Procedures}

Study staff engaged patients in the primary-care settings to solicit their participation. Those agreeing to participate provided informed consent and filled out several 
questionnaires, including assessments of sociodemographic profile, psychological health, medical history, sleep health, and use of medications. Clinical data including body mass index (BMI), blood pressure (BP), highdensity lipoprotein cholesterol (HDL), low-density lipoprotein cholesterol (LDL), and fasting plasma glucose (FPG) or hemoglobin (HbA1c) for those who have a diagnosis of diabetes were obtained from HealthBridge.

\section{Measures}

\section{Metabolic Risk}

Metabolic syndrome was defined according to the National Heart, Lung and Blood Institute, and the American Heart Association guidelines (Table 1). According to the joint interim statement, patients are considered to have the metabolic syndrome when they present with at least three of the following conditions: hypertension, diabetes, obesity and dyslipidemia (Table 1).

\section{Sleep Health}

Obstructive Sleep Apnea (OSA) was ascertained via the Apnea Risk Evaluation System $\left(\mathrm{ARES}^{\mathrm{TM}}\right) .{ }^{17}$ As previously described, ${ }^{17}$ patients with an ARES $^{\mathrm{TM}}$ score $\geq 6$ were considered at risk for OSA. ${ }^{17}$ The ARES questionnaire has a sensitivity of 0.94 , specificity of 0.79 (based on a clinical cut-off of AHI > 5), positive predictive value of 0.91 , and negative predictive value of $0.86 .^{17,18}$ The questionnaire includes age, weight, height, neck circumference, report of comorbid illness, such as high blood pressure, heart disease, diabetes, stroke, depression, lung disease,

Table I NCEP ATP III Definition of the Metabolic Syndrome

\begin{tabular}{|l|l|}
\hline Characteristic & NCEP ATP III \\
\hline Hypertension & BP medication or BP $>130 / 85 \mathrm{~mm} / \mathrm{Hg}$ \\
\hline Dyslipidemia & $\begin{array}{l}\text { Plasma triglycerides }>150 \mathrm{mg} / \mathrm{dL} \text { or } \\
\mathrm{HDL} \text { cholesterol }<40 \mathrm{mg} / \mathrm{dL} \text { in men and }<50 \\
\mathrm{mg} / \mathrm{dL} \text { in women }\end{array}$ \\
\hline Obesity & $\begin{array}{l}\text { Waist circumference }>40 \text { inches in men and }> \\
35 \text { inches in women }\end{array}$ \\
\hline $\begin{array}{l}\text { Hyperglycemia } \\
\text { (Diabetes) }\end{array}$ & Fasting plasma glucose $>110 \mathrm{mg} / \mathrm{dL}^{*}$ \\
\hline $\begin{array}{l}\text { Requirements for } \\
\text { Diagnosis }\end{array}$ & Any 3 of the above disorders \\
\hline
\end{tabular}

Notes: Guidelines from the National Cholesterol Education Program (NCEP) for defining Metabolic Syndrome. Note: BP denotes blood pressure; HDL, high-density lipoprotein. *Fasting plasma glucose was recently updated to $100 \mathrm{mg} / \mathrm{dl}$ by the American Diabetes Association. insomnia, sleep medication, pain medication and the Epworth Sleepiness scale.

Sleep duration was derived from self-reported questionnaire asking participants the average hours of sleep they receive in a 24-hour period. We defined short sleep as sleep duration lasting less than 7 hours in a 24-hour period based. This was referenced to healthy sleep defined as sleep durations between 7 and 8 hours.

\section{Psychological Health}

Depression was assessed using the Center for Epidemiological Studies-Depression (CES-D) scale, which is a 20 -item questionnaire that asks individuals to rate how often over the past week they experienced symptoms associated with depression, such as restless sleep, poor appetite, and feeling lonely. Item responses range from 0 to $3(0=$ Rarely or None of the Time, $1=$ Some or Little of the Time, 2 = Moderately or Much of the time, $3=$ Most or Almost All the Time). Scores range from 0 to 60, with high scores indicating greater depressive symptoms. A CES-D score greater or equal to 16 was used to identify patients with clinically meaningful depressive symptoms. The CESD-20 has excellent internal consistency (Cronbach's $\alpha=$ 0.88-0.91), excellent test-retest reliability $(\mathrm{ICC}=0.87) .{ }^{19}$ It has demonstrated adequate validity in measuring mental health (Pearson's r=0.75) and good sensitivity of $80.0 \%$.

Anxiety was measured with the Beck Anxiety Inventory (BAI), which is a 21 -item questionnaire assessing anxiety symptoms such as "wobbliness in legs", "scared" and "fear of losing control". 20 Accordingly, respondents are asked to rate how much each of these symptoms bothered them in the past week, on a scale ranging from 0 (not at all) to 3 (severely, I could barely stand it). Scores range from 0 to 63 , with a score of 16 or higher indicating moderate to severe anxiety. The scale has been validated in psychiatric outpatients diagnosed with various anxiety and depressive disorders. ${ }^{21}$ The BAI has a high internal consistency (Cronbach's $\alpha=0.92$ ) and a testretest reliability over one week of $0.75 .^{20}$ Both the CES-D and BAI are scales that identify depressive and anxiety symptoms.

\section{Statistical Analysis}

Descriptive analyses were conducted to identify missing data, outliers, means, and standard deviations across all demographic and study variables. Differences in clinical measurements, medical history/medications, health risks, and psychological factors were contrasted based on age 
groupings $(\leq 45,45-65,65+)$, examining potential effects of sex (male vs female) and birthplace (US-born vs foreign-born) using ANOVA for continuous variables and Chi-squared tests for categorical variables. Preliminary analyses using Pearson and Spearman correlations were conducted to determine the unadjusted associations among the variables of interest (data not shown). Logistic regression models adjusted for several factors including sex, birthplace, smoking history, alcohol consumption, family income, sleep apnea risk, depression, and anxiety, were performed to evaluate the association between age and metabolic burden. Due to missingness in smoking history, alcohol consumption, and family income, we conducted multiple imputation procedure with 100 times and aggregated point estimates as well as standard errors using R-package "mice". Specifically, each of the major components of metabolic burden was evaluated separately. The odds ratios (OR) along with $95 \%$ confidence interval (CI) were provided to quantify the association between factors of interest and each metabolic component (obesity, hypertension, diabetes, and dyslipidemia); p-value lower than 0.05 was considered statistically significant. Statistical analyses were performed with R 3.6.1 (http://www.r-pro ject.org).

\section{Results}

The present analysis was based on data from 1035 Blacks with metabolic syndrome in Brooklyn, NY (30 participants were excluded because of incomplete data). The age of the participants averaged $62 \pm 14$ years (range: $20-97$ ); 70\% were female and $60 \%$ were foreign-born Blacks. Overall, $43 \%$ reported an annual income lower than $\$ 10 \mathrm{~K}$. Of the sample, 93\% were diagnosed with hypertension; $61 \%$, diabetes; $72 \%$, dyslipidemia; $90 \%$ were overweight/ obese; $33 \%$ had a history of heart disease and 10\% a history stroke. According to ARES data, $48 \%$ of the participants were at risk for sleep apnea.

As illustrated in Table 2, compared with younger Blacks, those in the oldest age group were also characterized by higher level of health risks and co-morbidities (outside of metabolic conditions). Older Blacks had a higher rate of heart disease, cancer, arthritis, and were more likely to report use of antihypertensives, diuretics, insulin, statins, and cardiac medications. Conversely, they showed lower level depression and longer sleep duration overall. They were less likely to report a history of alcohol consumption, less likely to be at risk for sleep apnea, or to be a short sleeper.
Multivariate logistic regression analyses adjusting for sex, birthplace, smoking history, alcohol consumption, family income, depressive and anxiety symptoms, and sleep apnea risk demonstrated that age was a significant predictor of several metabolic components related to COVID-19, including hypertension ( $\mathrm{OR}=1.061 ; 95 \% \mathrm{CI}$ : $1.038-1.085 ; \mathrm{p}=0.001)$, diabetes $(\mathrm{OR}=1.028 ; 95 \% \mathrm{CI}$ : 1.015-1.041; $\mathrm{p}=0.001)$, and dyslipidemia $(\mathrm{OR}=0.976$; 95\% CI: $0.966-0.987 ; \mathrm{p}=0.001$ ), but not obesity. Analyses also indicated that age and obesity were associated in univariate analyses, but were no longer significant when covariates were adjusted in the model (Table 3).

\section{Discussion}

COVID-19 is a relatively new disease with sparse data regarding risk factors for severe diseases and mortality. Available data suggest that individuals with severe preexisting metabolic burden might be at higher risk for severe COVID-related diseases. ${ }^{1,2}$ Here, we analyzed data from the Metabolic Syndrome Outcome Study (MetSO), an NIH-funded study, to characterize the metabolic burden among Blacks in Brooklyn, one of the hardest hit boroughs in NYC, once the main epicenter of COVID19. ${ }^{14,16}$ Consistent with the observations from previous studies that Blacks in minority communities have been disproportionately affected by the adverse effects of the COVID-19 pandemic, ${ }^{2,3}$ the present analyses indicate a high level of metabolic burden among Blacks. Indeed, our data shows that $93 \%$ of Blacks participating in the study had a diagnosis of hypertension; $61 \%$ had diabetes, $72 \%$ had dyslipidemia, and $90 \%$ were overweight/obese. Our results suggest that metabolic syndrome, likely driven by the high prevalence of obesity, ${ }^{22}$ is a possible mechanism for the preponderance of COVID-19 infection and mortality burden among Blacks, presenting a possible avenue for system-level intervention to stem the rise of COVID-19 fatalities in Black communities.

Our main observation of extremely high metabolic burden among Blacks is consistent with recent findings from hospitalized patients with a COVID-19 diagnosis in the New York City area. In that study, enrolling 5700 patients the most common comorbidities were hypertension $(56.6 \%)$, obesity (41.7\%), and diabetes (33.8\%). ${ }^{1}$ Unfortunately, specific contrasts based on race/ethnicity was not provided, although it should be noted that $22.6 \%$ of the diagnosed cases represented individuals of the Black race/ethnicity. In the other large case series characterizing hospitalized patients with COVID-19 in NYC, 44.6\% had hypertension, 31.8\% had 
Table 2 Comparison of Medical History, Clinical, Psychological, Health, and Sleep Risk Factors, by Age Groupings

\begin{tabular}{|c|c|c|c|c|}
\hline Age & $\leq 45$ & 45 or $\leq 65$ & $>65$ & $p$-value \\
\hline $\mathbf{n}$ & 136 & 427 & 442 & \\
\hline \multicolumn{5}{|l|}{ Clinical Measures } \\
\hline Systolic Blood Pressure, mean (SD) & 132.72 (17.97) & I33.34 (|7.82) & I36.97 (16.58) & $0.003 * *$ \\
\hline Diastolic Blood Pressure, mean (SD) & 78.69 (II.5I) & $76.88(10.60)$ & 73.39 ( 11.38$)$ & $0.001 * * *$ \\
\hline Total cholesterol, mean (SD) & I80.29 (98.49) & I85.24 (48.6I) & 178.06 (45.22) & 0.184 \\
\hline HDL-C, mean (SD) & 43.06 (14.79) & 48.81 ( 17.28$)$ & $48.89(16.43)$ & $0.001 * * *$ \\
\hline Triglycerides, mean (SD) & $134.10(63.07)$ & 143.19 (91.17) & 130.17 (67.96) & 0.047 \\
\hline LDL-C, mean (SD) & $103.76(33.23)$ & $108.12(39.26)$ & $104.4 \mid(36.20)$ & 0.262 \\
\hline Glucose, mean (SD) & I35.67 (67.62) & $142.60(78.31)$ & I37.86 (75.66) & 0.530 \\
\hline Hemoglobin Alc, mean (SD) & $9.66(13.54)$ & $8.09(3.91)$ & $8.98(13.44)$ & 0.239 \\
\hline BMI, mean (SD) & $37.64(9.09)$ & $34.58(8.28)$ & $31.97(7.31)$ & $0.001 * * *$ \\
\hline Waist circumference, mean (SD) & $49.18(31.78)$ & $43.32(7.10)$ & $41.94(6.82)$ & $0.001 * * *$ \\
\hline \multicolumn{5}{|l|}{ Medical History and Medication Use } \\
\hline Hypertension (\%) & $108(87.1)$ & $391(92.4)$ & $430(97.3)$ & $0.001 * * *$ \\
\hline Dyslipidemia (\%) & $76(62.3)$ & $308(73.3)$ & $34 \mid(78.8)$ & $0.001 * * *$ \\
\hline Diabetes (\%) & $57(46.7)$ & $262(6 I .4)$ & $282(63.8)$ & $0.003 * *$ \\
\hline Heart Disease (\%) & $27(22.1)$ & $114(26.7)$ & $166(37.6)$ & $0.001 * * *$ \\
\hline Stroke $(\%)$ & II (8.9) & $33(7.7)$ & $44(10.0)$ & 0.514 \\
\hline Respiratory Problems (\%) & $26(21.7)$ & 91 (21.6) & $77(17.5)$ & 0.272 \\
\hline Cancer (\%) & $5(4.2)$ & $25(5.9)$ & $59(13.4)$ & $0.001 * * *$ \\
\hline Arthritis (\%) & $33(27.7)$ & $\mid 75(4 \mid .6)$ & $238(54.0)$ & $0.001 * * *$ \\
\hline Antihypertensives (\%) & $81(69.2)$ & $342(82.6)$ & $373(87.1)$ & $0.001 * * *$ \\
\hline Diuretics (\%) & $1.38(0.49)$ & $\mathrm{I} .43(0.50)$ & $1.50(0.50)$ & $0.034 *$ \\
\hline Insulin (\%) & $52(44.1)$ & $240(58.1)$ & $275(64.4)$ & $0.001 * * *$ \\
\hline Statins (\%) & $40(34.5)$ & $205(49.5)$ & $267(62.8)$ & $0.001 * * *$ \\
\hline Cardiac Drugs (\%) & $16(13.8)$ & $80(19.3)$ & $117(27.5)$ & $0.001 * * *$ \\
\hline Beta Blockers (\%) & $26(22.6)$ & $113(27.4)$ & $136(31.9)$ & 0.103 \\
\hline Antidepressants (\%) & $14(12.2)$ & $51(12.3)$ & $42(9.8)$ & 0.489 \\
\hline Major Tranquilizers (\%) & $5(4.3)$ & $21(5.1)$ & $14(3.3)$ & 0.432 \\
\hline Minor Tranquilizers (\%) & $2(1.7)$ & $21(5.1)$ & $9(2.1)$ & $0.034 *$ \\
\hline Sedative Hypnotics (\%) & $4(3.5)$ & II (2.7) & $10(2.3)$ & 0.785 \\
\hline \multicolumn{5}{|l|}{ Psychological Health and Sleep Factors } \\
\hline CESD, (mean (SD) & $19.02(8.22)$ & $17.60(7.21)$ & $16.38(6.56)$ & $0.001 * * *$ \\
\hline $\mathrm{BAI},($ mean $(\mathrm{SD})$ & $9.21(10.42)$ & $8.35(9.19)$ & $7.73(8.68)$ & 0.259 \\
\hline Sleep Duration, (mean (SD) & $6.21(2.11)$ & $5.84(1.92)$ & $6.27(1.82)$ & $0.003 * *$ \\
\hline Short Sleep (\%) & $90(66.2)$ & 307 (7I.9) & $262(59.3)$ & $0.001 * * *$ \\
\hline Long Sleep (\%) & $6(4.4)$ & $24(5.6)$ & $40(9.0)$ & 0.063 \\
\hline Insomnia (\%) & $12(10.2)$ & $5 I(12.1)$ & $38(8.8)$ & 0.288 \\
\hline Apnea Risk (\%) & $79(58.1)$ & $246(57.6)$ & $163(36.9)$ & $0.001 * * *$ \\
\hline Smoke History (\%) & $29(25.7)$ & $125(31.2)$ & $112(28.9)$ & 0.457 \\
\hline Alcohol Consumption (\%) & $32(29.6)$ & $89(23.1)$ & $64(17.1)$ & $0.022^{*}$ \\
\hline
\end{tabular}

Notes: Comparison of clinical measures, reported medical history and medication usage, and self-reported psychological measures, health risks, and sleep factors, based on age groupings for Blacks enrolled in the Metabolic Syndrome Outcomes study. p-values are from ANOVA (continuous variables) or chi-squared tests (categorical variables). *Note: BMI = body mass index; HDL-C = high-density lipoprotein cholesterol; LDL-C = low-density lipoprotein cholesterol; CESD = Center for Epidemiological Studies Depression scale; BAI = Beck Anxiety Inventory; Hypertension, Dyslipidemia, Diabetes, and Obesity defined in Table I. ${ }^{*} \mathrm{p}<0.05 ; * * \mathrm{p}<0.0 \mathrm{I} ; *^{* *} \mathrm{p}<0.00 \mathrm{I}$

diabetes, 25.9 had dyslipidemia, and $39.8 \%$ were obesity. ${ }^{23}$ As in the aforementioned study, specific racial/ethnic-based analyses were not provided, but it was reported that $14.5 \%$ of the patients were black. Additionally in a large cohort study of individuals in the UK that examined ethnic differences, individuals from non-White ethnicities had higher risk than those of the White ethnicity. Specifically, individuals of the Black ethnicity had an HR of 1.88 when age and sex were 
Table 3 Multivariate Logistic Regression of the Association Between Age and Metabolic Components Related to COVID-19

\begin{tabular}{|c|c|c|c|}
\hline & Odds Ratio & 95\% Confidence Interval & $p$-value \\
\hline \multicolumn{4}{|c|}{ Association of Age with Hypertension } \\
\hline Age & 1.061 & $1.038-1.085$ & $0.001 * * *$ \\
\hline Male Sex & 3.799 & $1.516-9.519$ & $0.004^{* *}$ \\
\hline Birthplace: US & 1.360 & $0.693-2.67 \mid$ & 0.372 \\
\hline Smoking History & 1.049 & $0.470-2.345$ & 0.906 \\
\hline Alcohol Consumption & 0.429 & $0.212-0.868$ & $0.019 *$ \\
\hline Income $\leq 10 \mathrm{~K}$ & 0.828 & $0.429-1.600$ & 0.575 \\
\hline CESD Score & 1.032 & $0.977-1.089$ & 0.265 \\
\hline BAI Score & 0.971 & $0.933-1.010$ & 0.140 \\
\hline Sleep Apnea Risk & 2.690 & $1.380-5.246$ & $0.004 * *$ \\
\hline \multicolumn{4}{|c|}{ Association of Age with Dyslipidemia } \\
\hline Age & 0.976 & $0.966-0.987$ & $0.001 * * *$ \\
\hline Male Sex & 0.477 & $0.354-0.643$ & $0.001 * * *$ \\
\hline Birthplace: US & 1.122 & $0.842-1.495$ & 0.433 \\
\hline Smoking History & 0.941 & $0.680-1.300$ & 0.711 \\
\hline Alcohol Consumption & 0.830 & $0.589-1.169$ & 0.287 \\
\hline Income $\leq 10 \mathrm{~K}$ & 1.026 & $0.765-1.375$ & 0.865 \\
\hline CESD Score & 0.995 & $0.972-1.019$ & 0.697 \\
\hline BAI Score & 0.994 & $0.975-1.012$ & 0.511 \\
\hline Sleep Apnea Risk & 1.397 & $1.059-1.844$ & $0.018^{*}$ \\
\hline \multicolumn{4}{|c|}{ Association of Age with Obesity } \\
\hline Age & 0.994 & $0.976-1.012$ & 0.517 \\
\hline Male Sex & 0.162 & $0.100-0.262$ & $0.001 * * *$ \\
\hline Birthplace: US & 1.457 & $0.886-2.394$ & 0.138 \\
\hline Smoking History & 0.573 & $0.344-0.955$ & $0.033^{*}$ \\
\hline Alcohol Consumption & 1.487 & $0.819-2.698$ & 0.192 \\
\hline Income $\leq$ IOK & 0.944 & $0.567-1.573$ & 0.825 \\
\hline CESD Score & 0.988 & $0.946-1.031$ & 0.568 \\
\hline BAI Score & 1.025 & $0.988-1.064$ & 0.183 \\
\hline Sleep Apnea Risk & 1.809 & $1.127-2.904$ & $0.014 *$ \\
\hline \multicolumn{4}{|c|}{ Association of Age with Diabetes } \\
\hline Age & 1.028 & $1.015-1.041$ & $0.001 * * *$ \\
\hline Male Sex & 1.229 & $0.838-1.802$ & 0.291 \\
\hline Birthplace: US & 0.712 & $0.502-1.010$ & 0.057 \\
\hline Smoking History & 1.241 & $0.821-1.878$ & 0.306 \\
\hline Alcohol Consumption & 0.983 & $0.644-1.498$ & 0.935 \\
\hline Income $\leq 10 \mathrm{~K}$ & $1.04 \mid$ & $0.727-|.49|$ & 0.827 \\
\hline CESD Score & 0.983 & $0.955-1.011$ & 0.233 \\
\hline BAI Score & 1.007 & $0.984-1.030$ & 0.552 \\
\hline Sleep Apnea Risk & 0.999 & $0.707-I .4 I I$ & 0.995 \\
\hline
\end{tabular}

Notes: Four separate multivariate logistic regression models each adjusted sex (male as reference), birthplace (US as reference), smoking history, alcohol consumption, depressive symptoms, anxiety symptoms, and sleep apnea risk to quantify the association between age and each metabolic component related to COVID-I9 (obesity, hypertension, diabetes, and dyslipidemia). ${ }^{*} p<0.05$; ${ }^{* *} p<0.01$; ${ }^{* * *} p<0.001$

Abbreviations: CESD, Center for Epidemiological Studies Depression scale; BAI, Beck Anxiety Inventory.

adjusted; of note, risks were further reduced $(\mathrm{HR}=1.43)$ after adjustment for age, sex, as well as comorbid disorders (including hypertension and diabetes) $)^{24}$ Therefore, while most published studies made no systematic racial/ethnic comparisons of the level of metabolic burden, it is apparent that Blacks with elevated burden, as observed in our study, would be at greater risk of exhibiting the cytokine release syndrome, commonly observed among patients who experience severe COVID-related complications. ${ }^{25,26}$ Conceivably, the disproportionate representation of Blacks in reported case 
fatalities could be a function of the greater metabolic burden they experience. ${ }^{1,23}$ Future analyses are required before any definitive explanations can be offered.

A consistent finding in the reported COVID-19 literature has been that generally older persons and men seem more likely to receive a diagnosis and experience more severe symptoms. ${ }^{1,23}$ Our analyses explored whether older Blacks could be at greater risk as a result of higher metabolic burden. In our sample, Blacks in the higher age groupings exhibited a greater metabolic burden, anchored by the presence of obesity, hypertension, diabetes, and dyslipidemia. Of interest, we also noted that older Blacks tended to have greater level of health risks and co-morbidities, outside of metabolic risk, as well. Multivariateadjusted regression modeling showed that the finding of greater age-associated metabolic burden was independent of individuals' sex, place of birth or other health risk factors. Except for obesity, which showed significant associations in univariate associations, age remained a significant predictor of the other three components of metabolic burden, after adjustment for all covariates in the models. Therefore, it is evident that while sex and place of birth are important factors in understanding level of metabolic burden, they did not attenuate the associations of age with hypertension, diabetes, and dyslipidemia.

Our results underscore the importance of considering the severity of pre-existing metabolic burden in various populations in classifying which strata may be at greater risk of COVID-19 diagnosis and mortality resulting from medical complications. These findings suggest that although older Blacks might be at greater risk, Blacks of younger ages are also vulnerable given that their level of metabolic burden is greater than that reported in previous studies characterizing patients hospitalized due to COVIDrelated medical complications. Our results also demonstrate that individuals' sex or place of birth, which is a crucial factor in understanding health profile of Brooklyn residents, did not substantially affect overall level of metabolic burden. We acknowledge that our present analyses were not based on Blacks presenting at the hospital for COVID-related symptoms. Moreover, these analyses do not provide a contrast with other relatively healthy Blacks, which would provide a comprehensive view of all Blacks in Brooklyn likely to be exposed to COVID19. Nonetheless, these findings offer an important context for understanding why Blacks tend to be disproportionately represented in published data on adverse effects of COVID-19. ${ }^{2}$ In line with the mandate to ensure greater health parity in all US communities, ${ }^{7-9}$ public health officials should envisage adoption of policies addressing equitable resource allocation strategies to reduce inequities related to metabolic risk for immediate implementation to combat the scourge of COVID-19 in underserved communities. ${ }^{27}$ Future studies should examine the likelihood of COVID-19 diagnosis and case fatality using a systematic approach enrolling Blacks with and without pre-existing metabolic burden.

\section{Abbreviations}

MetSO, Metabolic Syndrome Outcome Study; BMI, body mass index; BP, blood pressure; HDL, high-density lipoprotein cholesterol; LDL, low-density lipoprotein cholesterol; FPG, fasting plasma glucose; HbAlc, hemoglobin; OSA, obstructive sleep apnea; ARES ${ }^{\mathrm{TM}}$, Apnea Risk Evaluation System; CES-D, Center for Epidemiological Studies-Depression; BAI, Beck Anxiety Inventory.

\section{Data Sharing Statement}

The dataset utilized for analyses of the current study are available from the corresponding author upon reasonable request.

\section{Statement of Ethics}

All procedures performed in this study involving human participants were completed in accordance with the ethical standards of the NYU Langone and SUNY Downstate Medical Center Institutional Review Boards and with the Helsinki declaration and its amendments.

\section{Author Contributions}

GJ-L conceptualized and designed the study. PJ and ML processed and analyzed the data and prepared tables. AS helped to develop the scientific arguments and contributed to data interpretation. ADT contributed to the discussion and reviewed/edited the manuscript. All authors made substantial contributions to conception and design, acquisition of data, or analysis and interpretation of data; took part in drafting the article or revising it critically for important intellectual content; agreed to submit to the current journal; gave final approval of the version to be published; and agree to be accountable for all aspects of the work.

\section{Funding}

This research was supported by funding from the National Institutes of Health: R25HL105444, R01MD004113, R01AG056531, K07AG052685, R01HL152453, and 
R01HL142066. The funding source had no role in the design, conduct, or analysis of the study, or in the submission decision.

\section{Disclosure}

The authors have no conflicts of interest to declare for this work.

\section{References}

1. Richardson S, Hirsch JS, Narasimhan M, et al. Presenting characteristics, comorbidities, and outcomes among 5700 patients hospitalized with COVID-19 in the New York city area. JAMA. 2020;323:2052. doi:10.1001/jama.2020.6775

2. City of New York. City of New York. COVID-19: data. 2020. Available from: www1.nyc.gov/site/doh/covid/covid-19-data.page. Accessed April 25, 2020.

3. Centers for Disease Control and Prevention. Provisional death counts for coronavirus disease (COVID-19): weekly state-specific data updates by select demographic and geographic characteristics; 2020. Available from: www.cdc.gov/nchs/nvss/vsrr/covid_weekly. Accessed April 24, 2020.

4. New York City Department of Health ARL. The color of coronavirus: COVID-19 deaths by race and ethnicity in the U.S.; 2020. Available from: https://www.apmresearchlab.org/covid/deaths-by-race. Accessed May 11, 2020.

5. Institute of medicine committee on $\mathrm{U}$, eliminating $\mathrm{R}$, ethnic disparities in health C. In: Unequal Treatment: Confronting Racial and Ethnic Disparities in Health Care. Smedley BD, Stith AY, Nelson AR, eds. Washington (DC): National Academies Press (US) Copyright 2002 by the National Academy of Sciences. All rights reserved. 2003.

6. Williams DR, Mohammed SA. Discrimination and racial disparities in health: evidence and needed research. J Behav Med. 2009;32 (1):20-47.

7. Bibbins-Domingo K. This time must be different: disparities during the COVID-19 pandemic. Ann Intern Med. 2020;173:233-234. doi:10.7326/M20-2247

8. Chowkwanyun M, Reed AL Jr. Racial health disparities and Covid19 - caution and context. $N$ Engl J Med. 2020;383:201-203. doi:10.1056/NEJMp2012910

9. Laurencin CT, McClinton A. The COVID-19 pandemic: a call to action to identify and address racial and ethnic disparities. $J$ Racial Ethn Health Disparities. 2020;1-5.

10. Moore JX, Chaudhary N, Akinyemiju T. Metabolic syndrome prevalence by race/ethnicity and sex in the united states, national health and nutrition examination survey, 1988-2012. Prev Chronic Dis. 2017;14:E24E24. doi:10.5888/pcd14.160287

11. Clark LT, El-Atat F. Metabolic syndrome in African Americans: implications for preventing coronary heart disease. Clin Cardiol. 2007;30(4):161-164. doi:10.1002/clc.20003

12. Smith KW, Krieger N, Kosheleva A, et al. A structural model of social determinants of the metabolic syndrome. Ethn Dis. 2020;30 (2):331-338. doi:10.18865/ed.30.2.331
13. Williams NJ, Jean-Louis G, Brown CD, McFarlane SI, Boutin-Foster $\mathrm{C}$, Ogedegbe G. Telephone-delivered behavioral intervention among blacks with sleep apnea and metabolic syndrome: study protocol for a randomized controlled trial. Trials. 2014;15:225. doi:10.1186/17456215-15-225

14. Ramos AR, Wallace DM, Pandi-Perumal SR, et al. Associations between sleep disturbances and diabetes mellitus among blacks with metabolic syndrome: results from the metabolic syndrome outcome study (MetSO). Ann Med. 2015;47(3):233-237. doi:10.3109/ 07853890.2015 .1015601

15. Ravenell J, Seixas A, Rosenthal DM, et al. Effect of birthplace on cardiometabolic risk among blacks in the Metabolic Syndrome Outcome Study (MetSO). Diabetol Metab Syndr. 2016;8:14. doi:10.1186/s13098-016-0130-z

16. Williams NJ, Castor C, Seixas A, Ravenell J, Jean-Louis G. Sleep disorders and symptoms in blacks with metabolic syndrome: the Metabolic Syndrome Outcome Study (MetSO). Ethn Dis. 2018;28 (3):193-200. doi:10.18865/ed.28.3.193

17. Levendowski DJ, Morgan T, Montague J, Melzer V, Berka C, Westbrook PR. Prevalence of probable obstructive sleep apnea risk and severity in a population of dental patients. Sleep Breathing. 2008;12(4):303-309. doi:10.1007/s11325-008-0180-z

18. Enciso R, Clark GT. Comparing the Berlin and the ARES questionnaire to identify patients with obstructive sleep apnea in a dental setting. Sleep Breathing. 2011;15(1):83-89. doi:10.1007/s11325-0100328-5

19. Lewinsohn PM, Seeley JR, Roberts RE, Allen NB. Center for Epidemiologic Studies Depression Scale (CES-D) as a screening instrument for depression among community-residing older adults. Psychol Aging. 1997;12(2):277-287. doi:10.1037/0882-7974.12.2.277

20. Beck AT, Epstein N, Brown G, Steer RA. An inventory for measuring clinical anxiety: psychometric properties. J Consult Clin Psychol. 1988;56(6):893-897. doi:10.1037/0022-006X.56.6.893

21. Spitzer RL, Williams JB, Gibbon M, First MB. The Structured Clinical Interview for DSM-III-R (SCID). I: history, rationale, and description. Arch Gen Psychiatry. 1992;49(8):624-629. doi:10.1001/ archpsyc. 1992.01820080032005

22. Luzi L, Radaelli MG. Influenza and obesity: its odd relationship and the lessons for COVID-19 pandemic. Acta Diabetol. 2020;57(6):759764. doi:10.1007/s00592-020-01522-8

23. Petrilli CM, Jones SA, Yang J, et al. Factors associated with hospitalization and critical illness among 4103 patients with COVID-19 disease in New York City. medRxiv. 2020. 2020.2004.2008. 20057794..

24. Williamson EJ, Walker AJ, Bhaskaran K, et al. Factors associated with COVID-19-related death using OpenSAFELY. Nature. 2020;584:430-436. doi:10.1038/s41586-020-2521-4

25. Liu B, Li M, Zhou Z, Guan X, Xiang Y. Can we use interleukin-6 (IL-6) blockade for coronavirus disease 2019 (COVID-19)-induced cytokine release syndrome (CRS)? J Autoimmun. 2020;102452.

26. Pedersen SF, Ho YC. SARS-CoV-2: a storm is raging. $J$ Clin Invest. 2020;130(5):2202-2205. doi:10.1172/JCI137647

27. Brunton CT, Smedley BD. Building public health capacity to advance equity. JPHMP. 2019;25(4):411-412. doi:10.1097/PHH.00000000 00001040 
Diabetes, Metabolic Syndrome and Obesity: Targets and Therapy is an international, peer-reviewed open-access journal committed to the rapid publication of the latest laboratory and clinical findings in the fields of diabetes, metabolic syndrome and obesity research. Origina research, review, case reports, hypothesis formation, expert opinion and commentaries are all considered for publication. The manuscript management system is completely online and includes a very quick and fair peer-review system, which is all easy to use. Visit http://www.dovepress.com/testimonials.php to read real quotes from published authors.

Submit your manuscript here: https://www.dovepress.com/diabetes-metabolic-syndrome-and-obesity-targets-and-therapy-journal 